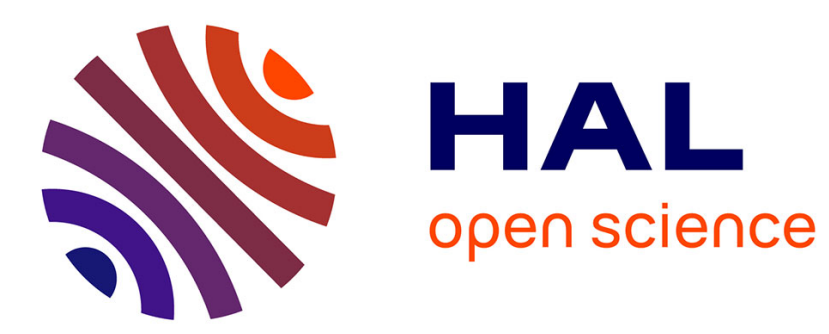

\title{
Efficiency of genomic selection in a purebred pig male line
}

\author{
Thierry Tribout, Catherine Larzul, Florence Phocas
}

\section{To cite this version:}

Thierry Tribout, Catherine Larzul, Florence Phocas. Efficiency of genomic selection in a purebred pig male line. Journal of Animal Science, 2012, 90 (12), pp.4164-4176. 10.2527/jas.2012-5107 . hal01001279

\section{HAL Id: hal-01001279 \\ https://hal.science/hal-01001279}

Submitted on 29 May 2020

HAL is a multi-disciplinary open access archive for the deposit and dissemination of scientific research documents, whether they are published or not. The documents may come from teaching and research institutions in France or abroad, or from public or private research centers.
L'archive ouverte pluridisciplinaire HAL, est destinée au dépôt et à la diffusion de documents scientifiques de niveau recherche, publiés ou non, émanant des établissements d'enseignement et de recherche français ou étrangers, des laboratoires publics ou privés. 


\section{JOURNAL OF ANIMAL SCIENCE}

The Premier Journal and Leading Source of New Knowledge and Perspective in Animal Science

\section{Efficiency of genomic selection in a purebred pig male line T. Tribout, C. Larzul and F. Phocas}

J ANIM SCI 2012, 90:4164-4176.

doi: 10.2527/jas.2012-5107 originally published online August 2, 2012

The online version of this article, along with updated information and services, is located on the World Wide Web at:

http://www.journalofanimalscience.org/content/90/12/4164 


\title{
Efficiency of genomic selection in a purebred pig male line ${ }^{1}$
}

\author{
T. Tribout, $* \dagger^{2}$ C. Larzul, $* \dagger$ and F. Phocas $* \dagger$ \\ *INRA, UMR1313 Génétique Animale et Biologie Intégrative, F-78350 Jouy-en-Josas, France; \\ and †AgroParisTech, UMR1313 Génétique Animale et Biologie Intégrative, F-75231 Paris, France
}

\begin{abstract}
Stochastic simulation was used to compare the efficiency of 3 pig breeding schemes based on either traditional genetic evaluation or genomic evaluation. The simulated population contained 1,050 female and 50 male breeding animals. It was selected for $10 \mathrm{yr}$ for a synthetic breeding goal that included 2 traits with equal economic weights and heritabilities of 0.2 or 0.4 . The reference breeding scheme, named BLUP-AM, was based on the phenotyping of all candidates $(13,770$ animals/yr) for Trait 1 and of relatives from $10 \%$ of the litters (270 animals/yr) for Trait 2 and on BLUP-Animal Model genetic evaluations. Under the first alternative scenario, named GE-1TP, selection was based on genomic breeding values (GBV) estimated with one training population (TP) made up of candidate relatives phenotyped for both traits, with a size increasing from 1,000 to 3,430 over time. Under the second alternative scenario, named GE-2TP, the GBV for Trait 2 were estimated using a TP identical to that of GE-1TP, but the GBV for Trait 1 were estimated using a large TP made up of candidates that increased in number from 13,770 to 55,080 over time. Over the simulated period, both genomic breeding schemes generated 39 to $58 \%$ more accurate EBV for Trait 2 than the reference scheme,
\end{abstract}

resulting in 78 to $128 \%$ (GE-1TP) and 63 to $84 \%$ (GE2TP) greater average annual genetic trends for this trait. For Trait 1, GE-1TP was 18 to $24 \%$ less accurate than BLUP-AM, reducing average annual genetic trends by 27 to $44 \%$. By contrast, GE-2TP generated 35 to $43 \%$ more accurate EBV and 8 to $22 \%$ greater average annual genetic trends for Trait 1 than the reference scheme. Consequently, GE-2TP was 27 to $33 \%$ more efficient in improving the global breeding goal than BLUP-AM whereas GE-1TP was globally as efficient as the reference scheme. Both genomic schemes reduced the inbreeding rate, the greatest decrease being observed for GE-2TP ( -49 to $-60 \%$ compared with BLUP-AM). In conclusion, genomic selection could substantially and durably improve the efficiency of pig breeding schemes in terms of reliability, genetic trends, and inbreeding rate without any need to modify their current structure. Even though it only generates a small TP, limited annual phenotyping capacity for traits currently only recorded on relatives would not be prohibitive. A large TP is, however, required to outperform the current schemes for traits recorded on the candidates in the latter.

Key words: breeding scheme, genomic selection, pig, production traits, simulation

(C) 2012 American Society of Animal Science. All rights reserved.

J. Anim. Sci. 2012.90:4164-4176 doi:10.2527/jas2012-5107

\section{INTRODUCTION}

Genomic selection (GS) has successfully been implemented for dairy cattle (e.g., in Holstein, Jersey,

\footnotetext{
${ }^{1}$ The authors acknowledge A. Legarra for providing the program used for the GBLUP evaluations and I. Misztal for supplying the BLUPF90 software. The authors would also like to thank J.C.M. Dekkers for his valuable advices. The authors wish to thank the ANR for financial support (ANR_10_GENOM_BTV_015).

${ }^{2}$ Corresponding author: thierry.tribout@jouy.inra.fr

Received January 10, 2012.

Accepted June 19, 2012.
}

and Brown-Swiss populations; Hayes et al., 2009). Compared with traditional schemes based on progeny testing, GS enables earlier accurate selection from a larger number of candidates. The ensuing shorter generation intervals and increased selection intensities have resulted in markedly greater annual genetic trends.

Such an improvement is tempting in other species. Implementation of GS can be envisaged in pigs since the availability of the SNP60 Illumina BeadChip (Illumina, Inc., San Diego, CA). However, current pig breeding schemes are characterized by high selection 
intensities and very short generation intervals. Therefore, the impact of GS on these 2 characteristics is expected to be small. However, accuracy of EBV is generally poor in pigs, and GS could potentially improve scheme efficiency through this parameter (Muir, 2007).

Therefore, the benefits of GS should be evaluated carefully before any implementation. Lillehammer et al. (2011) showed that GS could increase the genetic gain in dam lines for traits only recorded on females. Nevertheless, no other study based on a pig nucleus structure has been conducted.

Most pig male line breeding schemes are based on the on-farm phenotyping of candidates for a few fattening traits and on phenotyping in testing stations of a limited number of relatives or candidates for traits that are expensive or difficult to measure. The accuracy of EBV is particularly low for these latter traits, resulting in limited genetic trends, and GS might increase selection efficiency. Nevertheless, the limited phenotyping capacity might not allow for the establishment of an adequate training population (TP) for these traits.

The objective of the present study was, therefore, to compare, in terms of genetic trends, accuracy, and inbreeding, the efficiency of a current pig male line breeding scheme based on the combined phenotyping of candidates and relatives with alternative schemes based on GS.

\section{MATERIALS AND METHODS}

The comparison of the efficiency of different breeding schemes was ensured by the stochastic simulation of a purebred paternal pig population made up of 1,050 breeding females and 50 breeding males, with a family structure similar to the French Piétrain breed. The simulated population was selected for a synthetic breeding goal that included 2 traits with equal economic weights per genetic SD. Trait 1 represents one of the fattening traits that are cheap and easy to record on farm on a large number of candidates (e.g., growth rate, ultrasonic backfat thickness, or loin depth) whereas Trait 2 represents a trait that is too difficult or too expensive to measure on a large scale (e.g., meat quality, feed efficiency, and intramuscular fat).

Three breeding scenarios were considered in this study, differing in terms of phenotyping procedures and breeding value estimations. Under the first scenario, named BLUP-AM and representing a current traditional male line breeding scheme, the breeding value estimation was based on a conventional BLUP-Animal Model (Quaas and Pollak, 1980), the phenotypes for Trait 1 being recorded on the candidates, and the phenotypes for Trait 2 being recorded on a small number of relatives. Under the second scenario, named GE-1TP, the candidates were not phenotyped and selection decisions were based on genomic estimated breeding values (GEBV; Meuwissen et al., 2001), which were estimated using a single TP that was genotyped and phenotyped for both traits and only comprised relatives of the candidates. Under the third scenario, named GE-2TP, Trait 1 was recorded on the candidates and Trait 2 was recorded on their relatives as in the BLUP-AM scenario, but the former and latter animals constituted 2 distinct TP to estimate the GEBV for Trait 1 (with a large TP) and for Trait 2 (with a small $\mathrm{TP})$, respectively.

Each replicate of the simulation consisted in 2 successive main steps: i) creation of a base population before selection presenting a linkage disequilibrium (LD) of a desired level and ii) simulation of the genetic evolution of the population under various phenotyping and selection procedures.

\section{Generation of the Base Population}

At the beginning of each replicate, a base population of 400 males and 400 females was created. Each animal had 10 pairs of $100-\mathrm{cM}$ chromosomes, each of them carrying 3,600 equally spaced biallelic loci that might become SNP or QTL thereafter. In Generation 0, the genotype of each animal for each locus was randomly sampled from a Bernoulli distribution with frequency of 0.5 . Recombination was modeled by sampling in a binomial function the number of chromosomal intervals (constituted by the segment between 2 adjacent loci) where crossing over occurred during gametogenesis and then sampling the locations of these intervals under a uniform distribution. No mutation process was implemented.

The 400 females were randomly mated to one of the 400 males for 350 generations, each female producing 1 male offspring and 1 female offspring, and the new generation completely replacing the previous one. After 350 generations, a bottleneck was created by reducing the population size to 45 males and 45 females. The population was randomly mated for the next 35 generations with complete renewal at each generation and was then extended to 1,200 females and 400 males for the next 3 generations.

The number of random generations simulated, and the evolution of the size of the base population over generations of mating were determined in order to produce a population presenting a short- and long-range LD structure comparable to the LD structure actually observed in French pig populations (Figure 1; S. Boitard, INRA, Toulouse, France, personal communication).

In the last generation, 1,500 loci with a minor allele frequency above 0.05 were randomly sampled on each chromosome to be SNP markers that would be used for subsequent genomic evaluation procedures. Consequently, the SNP were unevenly spaced, the 
minimum distance between 2 adjacent markers being 28 $\mathrm{kb}$. The average distance was $67 \mathrm{~kb}$, corresponding to the average distance between adjacent markers on the porcineSNP60 Illumina BeadChip (Illumina, Inc.) after the usual elimination of noninformative markers and markers with technical problems. Among the remaining loci with a minor allele frequency above $0.05,2$ series of $\mathrm{n}$ loci were randomly sampled on each chromosome to be QTL, one series being QTL for Trait 1, the other series being QTL for Trait 2. Values of $\mathrm{n}$ were 10, 30, or 60 , depending on the QTL density considered in the simulations. To summarize, each of the 10 pairs of $100-$ cM chromosomes carried 1,500 unevenly spaced SNP, $n$ QTL for Trait 1 and n QTL for Trait 2.

By convention, the allele 1 of each QTL had no effect. A gamma distribution with shape and scale parameters equal to 0.4 and 1/1.66 (Hayes and Goddard, 2001) was used to sample the absolute value of effects of allele 2 of the QTL. The sign of the effect of allele 2 was positive or negative, with a probability of 0.5 . For both traits, the effects of the QTL were rescaled to result in a genetic variance of 1 . In the following, the true breeding value (TBV) $\mathrm{TBV}_{t_{i}}$ of animal $i$ for trait ( 1 or 2) was calculated as

$$
\mathrm{TBV}_{t_{i}}=\sum_{j=1}^{10}\left(\sum_{k=1}^{n} g_{t_{j k i}} \times q_{t_{j k i}}\right),
$$

in which $n$ is the number of QTL carried by each of the 10 chromosomes for each trait, $g_{t_{j k i}}$ is the genotype (i.e., 0,1 , or 2 copies of allele 2 ) of animal $i$ for the th QTL for trait on chromosome, and $q_{t_{j k i}}$ is the effect of the th QTL for trait on chromosome .

\section{Breeding Scenarios}

The second step in each replicate of the simulation started with the random sampling of 1,050 females and 50 males from the 1,200 females and 400 males of the last generation of the base population before selection to constitute the founders of the selected population. The 1,050

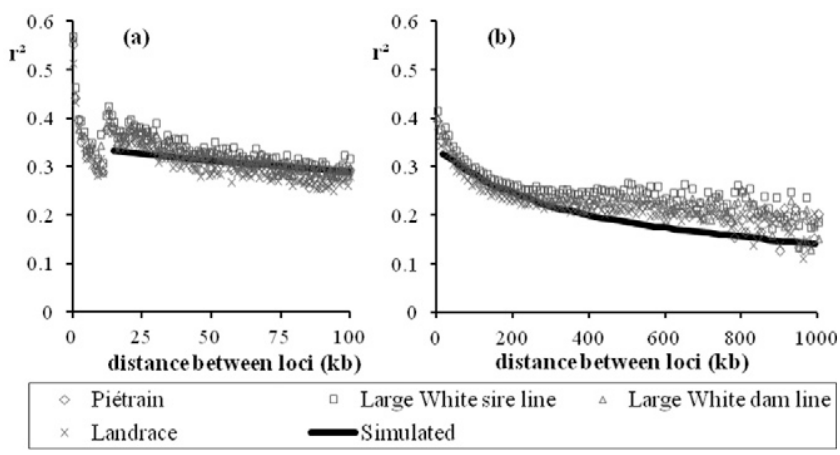

Figure 1. Linkage disequilibrium (r) observed in the 4 collective French pig breeds (S. Boitard, personal communication) and simulated in the present study for 0 - to $100-\mathrm{kb}$ intervals (a) and 0 - to 1,000-kb intervals (b), assuming $1 \mathrm{Mb} / \mathrm{cM}$. females were randomly assigned to 5 herds of equal size, and within each herd into 7 batches of 30 females. To boot the system, each of the 1,050 females was mated randomly to one of the 50 boars at the beginning of the replicate.

The demographic and breeding characteristics of the simulated population are summarized in Figure 2, and were modeled on the average statistics of the French Pi train nucleus population, established from the database used for national genetic evaluations.

The time step (TS) in our simulations was $3 \mathrm{wk}$, which corresponds to the period elapsing between the farrowing of females from 2 consecutive batches. Consequently, 1 yr of simulation represented $18 \mathrm{TS}$, and a sow farrowed every $7 \mathrm{TS}$ until culling. At each TS, several tasks were performed within each herd.

Farrowing of the Batch(0) Sows and Birth of the Piglets. The 30 sows of the current batch, Batch $(0)$, farrowed. Three litters from each herd (i.e., 10 of the litters born) were randomly sampled and left out to simulate the various une pectedevents that can cause some litters to be unsuitable for genetic purposes (e.g., abortion or misidenti cation of the mated boar). All of the 0 remaining litters (i.e., 27 litters per herd) contained 3 female candidates. Among these 27 litters, the best 0 were selected according to the mean BV of their parents (i.e., 24 litters per herd) and each contained 3 male candidates. ach male and female candidate $i$ received a simulated record $y_{1}$ for Trait 1 available $8 \mathrm{TS}$ after its birth (i.e., when the animal is 5.5 mo old, corresponding to the end of the on-farm test period when selection decisions are made), simulated as $y_{1_{i}}=\mathrm{TBV}_{1_{i}}+B L_{1_{z}}+e_{1_{i}}$, in which $B L_{1_{z}}$ is the random environmental effect of the th litter in which animal $i$ is born, sampled from a normal distribution with variance

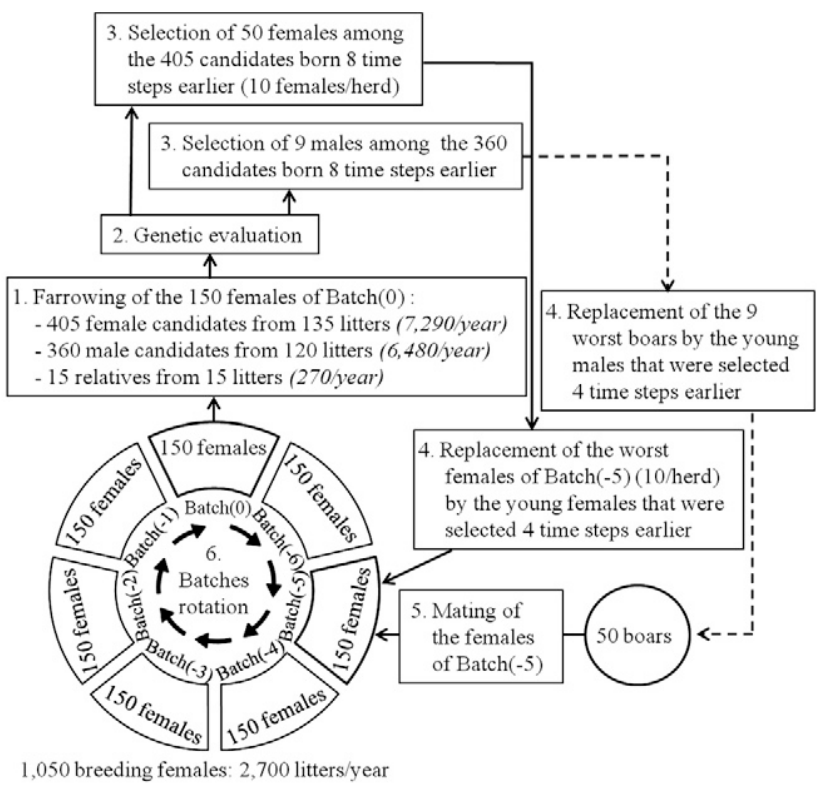

Figure 2. Population structure of the simulated breeding scheme and chronological order of events occurring at each simulated time step. 
$\sigma_{B L}^{2}=0.2, e_{1_{i}}$ is a random residual sampled from a normal distribution with variance $\sigma_{e_{1}}^{2}=\left(1 / h_{1}^{2}\right)-1-\sigma_{B L}^{2}, h_{1}^{2}$ being the heritability of Trait 1 , and assuming a genetic variance $\sigma_{a_{1}}^{2}$ of 1 .

Ten percent of the litters born (i.e., a total of 15 litters per batch in all 5 herds) also contained 1 piglet destined to be phenotyped for Traits 1 and 2 (these animals are referred to as "relatives" below). These $10 \%$ litters were chosen from the litters suitable for genetic purposes by selecting the best litters as a function of their parental EBV, giving priority to the litters of boars with the fewest offspring among "relatives" and balancing the number of litters chosen in each herd. As for the candidates, the simulated records $y_{1}$ and $y_{2}$ for Traits 1 and 2 were available 8 TS after the birth of "relative" $i$, and $y_{2_{i}}$ was simulated as $y_{2_{i}}=\mathrm{TBV}_{2_{i}}+e_{2_{i}}$, in which $e_{2_{i}}$ is a random residual sampled from a normal distribution with variance $\sigma_{e_{2}}^{2}=\left(1-h_{2}^{2}\right) / h_{2}^{2}$, $h_{2}^{2}$ being the heritability of Trait 2 and assuming a genetic variance $\sigma_{a_{2}}^{2}$ of 1 .

Breeding Value Estimation. Starting from the 26th TS (TS26; to allow time to accumulate sufficient phenotypes for Trait 2), breeding values for Trait 1 and Trait 2 (BLUP_EBV 1 and BLUP_EBV 2 ) were estimated for the complete population at each TS using a standard BLUP-Animal Model procedure and the BLUPF90 software (http://nce.ads.uga.edu/ ignacy/newprograms. $\mathrm{html}$ ), considering all the phenotypes and pedigrees recorded since creation of the herds (the most recent available phenotypes being recorded on candidates and relatives born 8 TS earlier). The genetic parameters were the same as those used to simulate the data, and the 2 traits were assumed to be genetically independent. For each animal $i$, the BLUP_EBV for Traits 1 and 2 were then combined in a global EBV (GL_EBV) giving equal weight to both traits as

\section{$\mathrm{GL}_{2} \mathrm{EBV}_{i}=1 \times$ BLUP_EBV $_{1_{i}}+1 \times$ BLUP_EBV $_{2_{i}}$.}

Selection Among Candidates. In each herd, the 10 best female candidates as a function of their GL_EBV were selected from the candidates in the herd available to renew the selection nucleus, after randomly eliminating $20 \%$ of the 81 candidates to simulate the uncontrolled culling events that can occur in real life and reduce selection efficiency (e.g., disease or leg problems). Similarly, the 9 best male candidates from the 360 candidates in the 5 herds were selected (a 20\% random loss of candidates was also applied) to renew the pool of boars. These selected animals would be available for reproduction 4 TS later. No attention was paid to relationships during the selection procedure.

Culling of Active Boars and Sows. In each herd, the worst third of the 30 sows in batch Batch( $(-5)$, according to their GL_EBV, were culled and replaced by the 10 young females that had been selected 4 TS earlier. Similarly, the 9 worst active boars were replaced by the 9 young males that had been selected 4 TS earlier.

Mating. In each herd, the 30 females in batch Batch $(-5)$ were randomly mated to 1 of the 50 available boars. Kinship relationships between males and females were not taken into account, which could thus lead to sib or half-sib matings.

Each TS ended with moving the active sows from their current batch to the next batch to prepare the next TS. Each task was repeated for 89 TS (i.e., 5 yr of BLUPAM selection after creation of the herds).

At TS90, the 3 scenarios diverged and were run for 180 additional TS (i.e., $10 \mathrm{yr}$ ). Under the BLUPAM scenario, the above procedure was executed 180 more times (i.e., 10 more yr). The 2 genomic scenarios considered in the study only differed from the BLUPAM scenario in terms of the procedure used to estimate breeding values. The number of candidates, phenotyping capacity for Trait 2 , and selection rates among candidates and breeding males and females were the same under all 3 scenarios.

Simulation of Training Populations. The accuracy of GS can be expressed as (Daetwyler et al., 2008; Meuwissen, 2009)

$$
r=\left[n \mathrm{~h}^{2} /\left(n \mathrm{~h}^{2}+4 N_{e} L \nu\right)\right]^{1 / 2},
$$

in which $\mathrm{n}$ is the number of individuals in the TP, $\mathrm{h}^{2}$ is the heritability of the trait, $L$ is the genome size (in Morgan), $N_{e}$ is the effective size of the population, and $4 N_{o} L \nu$ is the effective number of QTL loci in the genome. Consequently, considering constant SNP and QTL densities in all chromosomes of the genome, the number of phenotypes in the TP required to achieve the same accuracy of predicting breeding values when simulating a $\mathrm{K}$ Morgan genome rather than a 30 Morgan true porcine genome must be 30/K times smaller (Meuwissen, 2009). Because the genome simulated in this study was approximately one-third the actual pig genome (10 Morgan instead of 30 Morgan), the same ratio (1:3) was applied when simulating the TP to produce GEBV with appropriate accuracies.

To illustrate the relevance of Eq. [1], independently from the main study we simulated $5 \mathrm{yr}$ of the BLUP-AM scenario (TS1 to TS89) followed by $3 \mathrm{yr}$ of the GE-2TP scenario (TS90 to TS143), using the same population parameters as described above. Three situations were considered: i) 5 pairs of 100 -cM chromosomes applying a scaling factor of $1 / 6$ (Sit1a), ii) 10 pairs of $100-\mathrm{cM}$ chromosomes applying a scaling factor of $1 / 3$ (Sit2), and iii) 5 pairs of $100-\mathrm{cM}$ chromosomes applying an inadequate scaling factor of $1 / 3$ (Sit1b). Because the only 
concern regarding this illustration of Eq. [1] was the effect of the scaling factor on the accuracy of genomic evaluation, culling and replacements were random between TS1 and TS143, so that genetic variance was maintained as being equal to 1 on average for both traits in the 3 alternative situations. The results were averaged over 30 replicates.

GE-1TP Scenario. Under the GE-1TP scenario, male and female candidates were no longer phenotyped for Trait 1 after TS90. After that time, all candidates and breeding animals were genotyped, and all selection and culling decisions were based on GEBV, estimated as follows. At TS90, the effect of each of the 15,000 SNP on Trait 1 and Trait 2 was estimated using the BLUP methodology described by Meuwissen et al. (2001) on a TP of 1,000 animals, genotyped for the 15,000 SNP and phenotyped for Traits 1 and 2. This TP was made up of relatives of the candidates born between TS16 and TS82; in practice, according to the scaling argument described above, one-third of the relatives were randomly sampled at each TS to constitute the TP. The estimated SNP effects were used during the next 18 TS (i.e., 1 yr) to calculate the genomic $\mathrm{GEBV}_{t_{i}}$ of the breeding animals and candidates for Trait $t$ as

$$
\operatorname{GEBV}_{t_{i}}=\sum_{j=1}^{10}\left(\sum_{k=1}^{1500} g_{t_{j k i}} \times m_{t_{j k i}}\right),
$$

in which $g_{t_{j k i}}$ is the genotype (i.e., number of copies of allele 2) of animal $i$ for the $k$ th SNP on chromosome $j$ and $m_{t_{j k i}}$ is the estimated effect of the $k$ th SNP on chromosome $j$ for Trait $t$. As under the BLUP-AM scenario, GEBV for Trait 1 and 2 were combined as $\mathrm{GL}_{-} \mathrm{GEBV}_{i}=1 \times \mathrm{GEBV}_{1_{i}}+1 \times \mathrm{GEBV}_{2_{i}}$, and all selection decisions were based on the GL_GEBV ${ }_{i}$. Every year (i.e., in TS108, TS126, TS144, TS162, TS180, TS198, TS216, TS234, and TS252), the effects of the SNP on the traits were estimated again on the previous TP augmented by the 270 new animals (i.e., 90 simulated animals) phenotyped during the past year, and these new estimates were then used to calculate the GEBV ${ }_{t}$ of breeding animals and candidates for the next $18 \mathrm{TS}$.

GE-2TP Scenario. Under the GE-2TP scenario as with GE-1TP, all selection and culling decisions were based on GEBV, requiring all animals to be genotyped. The estimation procedure for GEBV for Trait 2 was the same as under the GE-1TP scenario, with a TP of 1,000 at TS90, increasing by 270 animals annually. Unlike the GE-1TP scenario, the male and female candidates were still phenotyped for Trait 1 after TS90, and consequently could be used to constitute and increase a TP for Trait 1 . As for Trait 2, an initial estimate of the SNP effects on Trait 1 was made using the BLUP methodology at TS90 with a TP of 13,770, corresponding to the male and female candidates phenotyped during the fifth yr of the simulation (TS73 to TS90); at each TS, one-third of the candidates were randomly sampled to constitute the TP. The TP for Trait 1 increased by 13,770 animals every year (i.e., 4,590 simulated animals) until TS144; after that, the oldest 13,770 animals were removed each year and replaced by the 13,770 most recent ones, so as to maintain the TP at a constant size of 55,080 animals (i.e., $4 \mathrm{yr}$ of candidate testing) and keep the computation time at a reasonable level.

For each scenario, 3 couples of medium and low heritabilities for Trait 1 and Trait 2 (0.4 and 0.4, 0.4 and 0.2 , and 0.2 and 0.2 referred to, respectively, as $\mathbf{M} \mathbf{M h}^{2}$, $\mathbf{M L h}{ }^{2}$, and $\mathbf{L L h} \mathbf{h}^{2}$ below) and 3 QTL densities (10 QTL/ Morgan per trait, 30 QTL/Morgan per trait, or 60 QTL/ Morgran per trait, which are referred to, respectively, as LowQd, MedQd, and HighQd) were considered at TS0. Nine parameter cases were thus simulated under each scenario.

For each TS, the mean and variance of the $\mathrm{TBV}_{1_{i}}, \mathrm{TBV}_{2_{i}}$ and $\mathrm{GL}_{-} \mathrm{TBV}_{i}=\mathrm{TBV}_{1_{i}}+\mathrm{TBV}_{2_{i}}$ values of candidates born in the TS considered as well as the mean of their inbreeding coefficients based on pedigree information were calculated to measure the genetic gain and the evolution of genetic variance and inbreeding over time. The correlation between the TBV and the BLUP or genomic EBV of candidates available for selection was also calculated at each TS to estimate the accuracy of the selection. All results were averaged over 200 replicates per simulated case.

\section{RESULTS}

\section{Relevance of the Scaling Relation Between the Length of the Simulated Genome and the Size of the Training Population}

Figure 3 presents the accuracy of the GEBV (i.e., correlation between TBV and GEBV) for the 765 candidates available for selection in each TS from TS90 to TS143, under the Sit1a, Sit2, and Sit1b situations. Overall, the time course of the accuracy of the GEBV for both traits under the 3 situations displayed similar profiles. The accuracy increased markedly every 18 TS (TS108 and TS128), and decreased slowly for the next 17 TS. The increases in accuracy corresponded to the yearly reestimation of the SNP effects considering a larger TP whereas the subsequent regular decreases reflected the progressive reduction in the degree of relationship between the population under evaluation and the TP. However, the consequences of the decreasing relationships between the candidates and the TP on the accuracy of the GEBV were small, and diminished from year to year. For example, the accuracy for Trait 1 decreased by $5.5,3.8$, and $3.3 \%$ during the first, second, and third years of selection, respectively. The presence of close relatives of the candidates in the TP became less and less important as TP size increased. 
Table 1. Reduction in genetic variance (\%) for Traits 1 and 2 under 3 scenarios during the last 10 yr simulated, according to the initial heritabilities of the traits and QTL densities ${ }^{1}$

\begin{tabular}{|c|c|c|c|c|c|c|c|}
\hline \multirow{4}{*}{$\begin{array}{l}\text { Initial } \\
\text { QTL } \\
\text { density }\end{array}$} & \multirow[b]{4}{*}{ Scenario $^{2}$} & \multicolumn{6}{|c|}{ Reduction in genetic variance for: } \\
\hline & & \multicolumn{3}{|c|}{ Trait 1 , genetic variance unit } & \multicolumn{3}{|c|}{ Trait 2, genetic variance unit } \\
\hline & & \multicolumn{6}{|c|}{ Initial heritabilities of traits ${ }^{3,4}$} \\
\hline & & $0.4 / 0.4$ & $0.4 / 0.2$ & $0.2 / 0.2$ & $0.4 / 0.4$ & $0.4 / 0.2$ & $0.2 / 0.2$ \\
\hline \multirow{3}{*}{$\begin{array}{l}\text { 10QTL/ } \\
\text { Morgan }\end{array}$} & BLUP-AM & -79 & -79 & -75 & -34 & -22 & -29 \\
\hline & GE-1TP & -64 & -67 & -59 & -67 & -53 & -60 \\
\hline & GE-2TP & -84 & -86 & -83 & -61 & -47 & -51 \\
\hline $\begin{array}{l}\text { 30QTL/ } \\
\text { Morgan }\end{array}$ & GE-2TP & -67 & -70 & -68 & -44 & -35 & -36 \\
\hline \multirow{3}{*}{$\begin{array}{l}\text { 60QTL/ } \\
\text { Morgan }\end{array}$} & BLUP-AM & -48 & -50 & -46 & -22 & -18 & -22 \\
\hline & GE-1TP & -41 & -44 & -41 & -47 & -41 & -48 \\
\hline & GE-2TP & -57 & -61 & -58 & -38 & -31 & -35 \\
\hline
\end{tabular}

\footnotetext{
${ }^{1}$ Results are averages of 200 replicates. composed of relatives for Trait 2 .

${ }^{3}$ Initial heritability for Trait 1 /initial heritability for Trait 2.

${ }^{4}$ For a given QTL density, all values within a column differ $(P<0.001)$.
}

${ }^{2}$ Simulated scenarios: BLUP-AM = breeding scheme based on traditional BLUP-Animal Model genetic evaluations using phenotypes of the candidates for Trait 1 and phenotypes of relatives for Trait 2; GE-1TP = breeding scheme based on genomic evaluations using a single training population composed of relatives; GE-2TP = breeding scheme based on genomic evaluations using a training population composed of candidates for Trait 1 and a training population

The accuracies of selection in Sit1a and Sit2, applying to the TP size a scaling factor proportional to the fraction of the length of the simulated genome, were close as expected from Eq. [1]. Conversely, the results for Sit1b showed that use of an inappropriate scaling factor (in Sit1b, the scaling factor was $1 / 3$ rather than $1 / 6)$ resulted in significantly different correlations $(P<$ 0.001 ) between the GEBV and true breeding values of the candidates. As a consequence, ignoring this scaling relation when simulating a genome shorter than the actual genome of the considered species would yield an overestimate of the accuracy of genomic evaluation and of the efficiency of GS because this expected response would actually require a larger TP.

\section{Comparison of the Efficiency of the Three Scenarios}

Overall, the relative results for the 3 scenarios from TS90 to TS270 in terms of genetic gain, accuracy, genetic variability, and inbreeding yielded the same general trends in the 9 combinations of heritabilities and QTL densities considered. Consequently, detailed results over time are only presented below for the $\mathrm{MLh}^{2}-$ HighQd parameter case, supplemented by tables of the time-averaged results per scenario for the 9 cases.

Genetic Variance. The genetic variance of the 2 traits decreased over time (Table 1) under all 3 scenarios. This decrease can be explained by the increase in frequency of favorable QTL alleles in the population due to selection. This decrease was even more rapid when the initial QTL density was less as shown in Figures $4 \mathrm{a}$ and $4 \mathrm{~b}$ for the 60 QTL/Morgan and 10 QTL/Morgan initial densities, respectively. An initial loss of genetic variance, particularly large for Trait 1, occurred during the first year after the implementation of selection (TS26 to TS44), its magnitude being consistent with the expected theoretical Bulmer effect (Dekkers, 1992) considering the applied selection intensities and the accuracy of the EBV for both traits. The decrease in genetic variance then continued gradually (from the slowest to the most rapid decrease: GE-1TP, BLUP-AM, and GE-2TP for Trait 1 and BLUP-AM, GE2TP, and GE-1TP for Trait 2).

Accuracy. The time course of the accuracy of EBV displayed different profiles under the 3 scenarios as shown in Figure 5 for the $\mathrm{MLh}^{2}$-HighQd parameter case. The accuracies under the BLUP-AM scenario decreased steadily from TS90 to TS270, with the rate of this decrease being slow for Trait 2 but more rapid for Trait 1 . This trend could be explained by the progressive decrease of genetic variance in the population: since the variance remained constant over time in the simulations, the proportion of the phenotypic variance of genetic origin decreased as did the ability to predict breeding values with a BLUP-AM evaluation. Because more data were available for Trait 1 than Trait 2 under the BLUP-AM scenario, selection was more efficient and the decrease in genetic variance and therefore in accuracy was more marked for Trait 1 (Figures 4 and 5).

Conversely, the accuracy of the genomic values estimated by considering the TP made up of relatives (i.e., Traits 1 and 2 under the GE-1TP scenario and Trait 2 under the GE-2TP scenario) increased globally over time in line with the size of the TP, but the rate of this increase gradually slowed down. The accuracy of the genomic values for Trait 1 under the GE-2TP scenario displayed a similar trend for as long as the number of candidates in the TP increased each year (i.e., until 


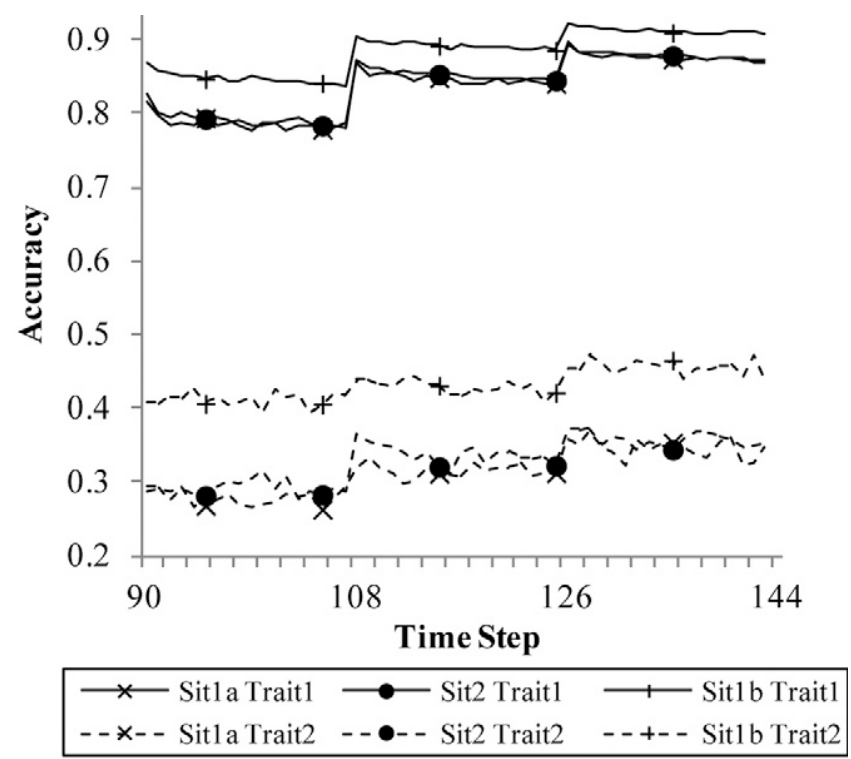

Figure 3. Evolution of the accuracy of genomic EBV of candidates available for selection at each time step, considering different lengths of simulated genome and different values for the scaling factor applied to the size of the training population. The initial QTL density was 30 QTL/M for each trait, and heritabilities of Trait 1 and Trait 2 were 0.4 and 0.2 , respectively. The results are averages of 30 replicates: Sit1a, 5 pairs of 100 -cM chromosomes applying a scaling factor of $1 / 6$; Sit 1 b, 5 pairs of 100 -cM chromosomes applying an inadequate scaling factor of 1/3; and Sit2, 10 pairs of 100 -cM chromosomes applying a scaling factor of $1 / 3$.

TS144), but decreased globally after that. A second annual pattern appeared alongside these general trends in all the cases; accuracies improved abruptly every year when SNP effects were reestimated on the updated TP and then decreased progressively for the next $17 \mathrm{TS}$.

The average accuracies from TS90 to TS270 are presented in Table 2 for the 3 scenarios. Both genomic scenarios produced significantly $(P<0.001)$ more accurate EBV for Trait 2 than the BLUP-AM scenario. Whereas the accuracy of the BLUP-AM EBV of candidates for selection was capped at 0.27 or 0.33 when the initial heritability of Trait 2 was 0.2 or 0.4 , respectively, the accuracy of GEBV rose to 0.36 $\left(h^{2}=0.2\right)$ or $0.46\left(h^{2}=0.4\right)$ as early as TS90 and then increased yearly with the addition of new animals to the TP (Figure 5). On average, between TS90 and TS270, the accuracy of GEBV for Trait 2 was about $50 \%$ greater than the accuracy of BLUP-AM EBV.

The 2 genomic scenarios generated divergent results for Trait 1. As for Trait 2, the GE-2TP scenario provided more accurate EBV for Trait $1(P<0.001)$ when compared with the BLUP-AM scenario. Accuracy increased from approximately 0.30 to 0.50 (for $\mathrm{h}^{2}$ of Trait $1=0.2$ ) and from approximately 0.40 to 0.60 (for $\mathrm{h}^{2}$ of Trait $1=0.4$ ) at TS90 when switching from the BLUP-AM evaluation to the genomic evaluation. On average, between TS90 and TS270, the accuracies of EBV for Trait 1 were 35 to $43 \%$ greater under the GE2TP scenario than under BLUP-AM. By contrast, the

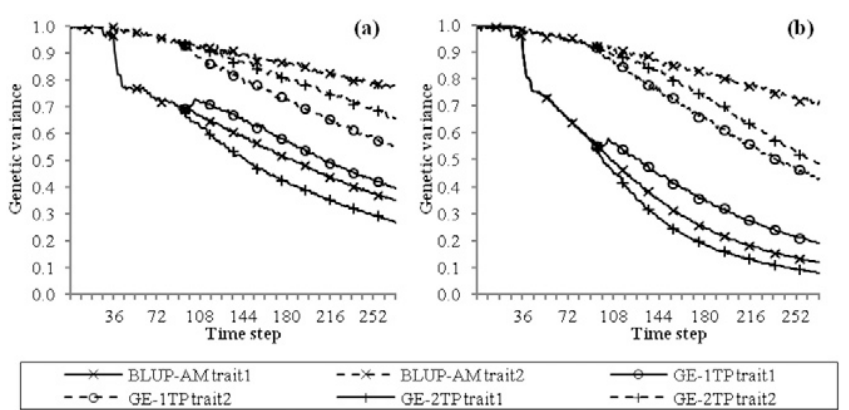

Figure 4. Evolution of genetic variance under 3 scenarios during the 15 yr simulated. Initial QTL density $=60$ QTL/M (a) or 10 QTL/M (b) for each trait, initial heritability of Trait $1=0.4$, and initial heritability of Trait $2=0.2$. The results are averages of 200 replicates. BLUP-AM = breeding scheme based on traditional BLUP-Animal Model genetic evaluations using phenotypes of the candidates for Trait 1 and phenotypes of relatives for Trait 2; GE-1TP = breeding scheme based on genomic evaluations using a single training population composed of relatives; GE-2TP = breeding scheme based on genomic evaluations using a training population composed of candidates for Trait 1 and a training population composed of relatives for Trait 2 .

accuracies of the EBV for Trait 1 were less $(P<0.001)$ under the GE-1TP scenario than under BLUP-AM at TS90 (e.g., 0.40 vs. 0.64 in the $\mathrm{MLh}^{2}$-HighQd case) when TP only contained 1,000 animals, and remained less until the end of the simulation, despite TP size increasing every year by 270 animals (Figure 5).

Genetic Trends. The above differences in genetic variances and in EBV accuracies yielded differences in terms of genetic trends between the 3 scenarios being compared (Table 3). Both genomic scenarios produced significantly $(P<0.001)$ greater average annual genetic trends for Trait 2 than the BLUP-AM scenario, this superiority tending to be greater for $\mathrm{MLh}^{2}$ than for $\mathrm{MMh}^{2}$ cases (i.e., for a lesser heritability of Trait 2). The average annual genetic trends between TS90 and TS270 were 78 to $128 \%$ greater under GE-1TP than under BLUP-AM, and 63 to $84 \%$ greater under GE-2TP than under BLUPAM. For example, in the $\mathrm{MLh}^{2}$-HighQd case, Trait 2 improved by $+0.13,+0.30$, and +0.24 genetic SD per year $\left(\boldsymbol{\sigma}_{g} / \mathbf{y r}\right)$ under the BLUP-AM, GE-1TP, and GE-2TP scenarios, respectively (Figure 6).

The results of the 2 genomic scenarios differed for Trait 1 . The annual genetic trends for this Trait were 27 to $44 \%$ less under the GE-1TP scenario than under BLUP-AM, this difference $(P<0.001)$ tending to be greater when the heritability of Trait 1 was less $\left(\mathrm{MLh}^{2}\right.$ case vs. $\mathrm{LLh}^{2}$ case). For example, in the $\mathrm{MLh}^{2}$-HighQd case, the genetic level of the population for Trait 1 increased by $+0.62 \sigma_{g} / \mathrm{yr}$ under the reference scenario, but only by $+0.42 \sigma_{g} / \mathrm{yr}$ under the GE-1TP scenario (Figure 6). Conversely, the GE-2TP scenario produced 8 to $22 \%$ greater average annual genetic trends for Trait 1 , this improvement being significant in all the cases considered $(P<0.001)$ and, as for Trait 2 , was greater with a smaller heritability of Trait 1 . 
As a consequence, scenario GE-2TP was globally 27 to $33 \%$ more efficient than scenario BLUP-AM in improving the global breeding goal (Trait $1+$ Trait 2). Despite the sampling variance of the results among the 200 replicates, the average TBV of the population at TS270 was greater under the GE-2TP scenario than under BLUP-AM in 97.8, 98.1, and $99.7 \%$ of the replicates for Trait 1, Trait 2, and the global breeding goal, respectively. By contrast, scenario GE-1TP was nearly as efficient as the reference scenario, its advantage for Trait 2 balancing out its poor result for Trait 1 .

Inbreeding. Both genomic scenarios resulted in a smaller global increase in the average inbreeding coefficient of the population than the BLUP-AM scenario (Table 4). Compared with the latter, average inbreeding at the end of the simulation was 13 to $30 \%$ less under the GE-1TP scenario and 49 to $60 \%$ lower under GE-2TP, depending on the initial QTL density and initial heritabilities considered. Nevertheless, whereas the GE-2TP and BLUP-AM scenarios displayed linear time trends for this parameter, the increase in the inbreeding coefficient was much more marked under the GE-1TP scenario during the first time period, before slowing down and stabilizing to approximately the same trend as that seen with GE-2TP (Figure 7). Therefore, as early as $3 \mathrm{yr}$ after the beginning of GS and up to the end of the simulated period, the inbreeding was about $30 \%$ less under the GE-2TP scenario than under GE-1TP.

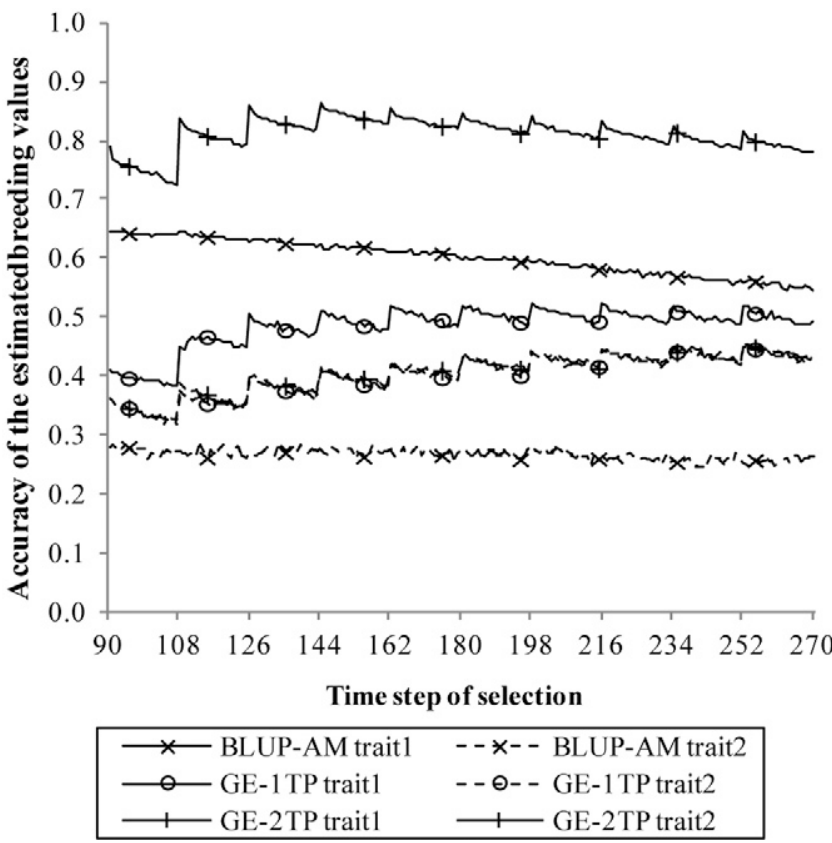

Figure 5. Accuracy of the EBV of candidates under 3 scenarios during the last $10 \mathrm{yr}$ simulated, with an initial QTL density of $60 \mathrm{QTL} / \mathrm{M}$ for each trait, initial heritability of Trait $1=0.4$, and initial heritability of Trait $2=0.2$. The results are averages of 200 replicates. BLUP-AM = breeding scheme based on traditional BLUP-Animal Model genetic evaluations using phenotypes of the candidates for Trait 1 and phenotypes of relatives for Trait 2; GE-1TP = breeding scheme based on genomic evaluations using a single training population composed of relatives; GE-2TP = breeding scheme based on genomic evaluations using a training population composed of candidates for Trait 1 and a training population composed of relatives for Trait 2 .

Table 2. Average accuracy of EBV of young candidates under 3 scenarios during the last $10 \mathrm{yr}$ of the simulated breeding schemes, according to the initial heritabilities of traits and QTL densities ${ }^{1}$

\begin{tabular}{|c|c|c|c|c|c|c|c|}
\hline \multirow{4}{*}{$\begin{array}{l}\text { Initial } \\
\text { QTL } \\
\text { density }\end{array}$} & \multirow[b]{4}{*}{ Scenario $^{2}$} & \multicolumn{6}{|c|}{ Average accuracy of EBV for: } \\
\hline & & \multicolumn{3}{|c|}{ Trait 1} & \multicolumn{3}{|c|}{ Trait 2} \\
\hline & & \multicolumn{6}{|c|}{ Initial heritabilities of traits ${ }^{3,4}$} \\
\hline & & $0.4 / 0.4$ & $0.4 / 0.2$ & $0.2 / 0.2$ & $0.4 / 0.4$ & $0.4 / 0.2$ & $0.2 / 0.2$ \\
\hline \multirow{3}{*}{$\begin{array}{l}\text { 10QTL/ } \\
\text { Morgan }\end{array}$} & BLUP-AM & 0.51 & 0.50 & 0.45 & $0.31^{\mathrm{a}}$ & $0.26^{\mathrm{a}}$ & 0.26 \\
\hline & GE-1TP & 0.42 & 0.40 & 0.34 & $0.46^{\mathrm{b}}$ & $0.37^{\mathrm{b}}$ & 0.37 \\
\hline & GE-2TP & 0.73 & 0.70 & 0.64 & $0.45^{\mathrm{b}}$ & $0.37^{\mathrm{b}}$ & 0.38 \\
\hline $\begin{array}{l}\text { 30QTL/ } \\
\text { Morgan }\end{array}$ & GE-2TP & 0.80 & 0.78 & 0.71 & $0.50^{\mathrm{b}}$ & $0.41^{\mathrm{b}}$ & $0.41^{\mathrm{b}}$ \\
\hline \multirow{3}{*}{$\begin{array}{l}60 \mathrm{QTL} / \\
\text { Morgan }\end{array}$} & BLUP-AM & 0.61 & 0.60 & 0.52 & 0.33 & $0.27^{\mathrm{a}}$ & 0.27 \\
\hline & GE-1TP & 0.49 & 0.48 & 0.40 & 0.51 & $0.40^{\mathrm{b}}$ & 0.41 \\
\hline & GE-2TP & 0.82 & 0.81 & 0.74 & 0.51 & $0.41^{\mathrm{b}}$ & 0.42 \\
\hline
\end{tabular}

\footnotetext{
${ }^{1}$ Results are averages of 200 replicates.

${ }^{2}$ Simulated scenarios: BLUP-AM = breeding scheme based on traditional BLUP-Animal Model genetic evaluations using phenotypes of the candidates for Trait 1 and phenotypes of relatives for Trait 2; GE-1TP = breeding scheme based on genomic evaluations using a single training population composed of relatives; GE-2TP = breeding scheme based on genomic evaluations using a training population composed of candidates for Trait 1 and a training population composed of relatives for Trait 2 .

${ }^{3}$ Initial heritability for Trait 1 /initial heritability for Trait 2.

${ }^{4}$ For a given QTL density, all values within a column differ $(P<0.001)$ except values with common superscripts.
} 


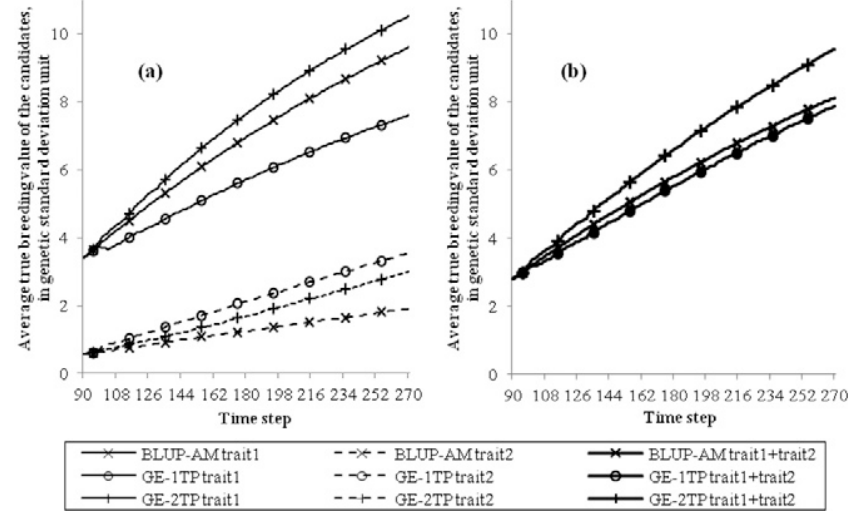

Figure 6. Realized genetic trends (in genetic SD units) for Traits 1 and 2 (a) and for the global breeding goal (Trait $1+$ Trait 2) (b) under 3 scenarios during the last $10 \mathrm{yr}$ simulated, with an initial QTL density of 60 QTL/M for each trait, initial heritability of Trait $1=0.4$, and initial heritability of Trait $2=0.2$. The results are averages of 200 replicates. BLUP-AM $=$ breeding scheme based on traditional BLUP-Animal Model genetic evaluations using phenotypes of the candidates for Trait 1 and phenotypes of relatives for Trait 2; GE-1TP = breeding scheme based on genomic evaluations using a single training population composed of relatives; $\mathrm{GE}-2 \mathrm{TP}=$ breeding scheme based on genomic evaluations using a training population composed of candidates for Trait 1 and a training population composed of relatives for Trait 2 .

\section{DISCUSSION}

The representativeness of stochastic simulation results compared with the results of real breeding schemes is obviously dependent on the quality of modeling. Attention was paid in this study to simulating a population with a realistic structure already selected for several years when the 3 scenarios started to diverge, and the use of adequate short- and long-range LD structure and marker density. However, some other assumptions were made that might have departed from reality. For instance, because the actual number of genes affecting a trait in a population is unknown, 3 arbitrary QTL densities were considered, but they nonetheless generated consistent results on the relative efficiencies of the 3 scenarios. Furthermore, the management of inbreeding was not considered in the selection and mating procedures whereas inbreeding is a major concern for pig breeders. Also, simplifications were made in the genomic evaluation procedures to keep the computation time reasonable. In particular, the yearly renewal of the TP and SNP effects reestimation yielded slightly smaller accuracies of GEBV than those that would have been obtained if the phenotypes of the candidates had been added to the TP at every TS. All these assumptions may have affected the results, which should therefore be considered as trends rather than precise predictions.

The results of this study tend to show that GS could substantially and durably increase the genetic gains achieved in a purebred pig male line based on the combined phenotyping of candidates and relatives for lowly to moderately heritable traits while significantly reducing the annual increase in inbreeding in the population without it being necessary to modify the structure of the current breeding scheme. In particular, although it may result in a small TP of limited power, a small annual phenotyping capacity for traits only recorded on relatives may be sufficient to implement a genomic scheme more efficient than the current breeding scheme based on BLUP-AM evaluation, the accuracy of which is poor for these traits. This situation is quite different from that of dairy cattle breeding schemes where a TP of several thousand bulls is needed to achieve the same high degree of accuracy as conventional progeny testing (Hayes et al., 2009). In the current study, the initial TP for Trait 2 under the GE-1TP and

Table 3. Average realized annual genetic trends under 3 scenarios during the last $10 \mathrm{yr}$ simulated, according to the initial parameter values for the heritabilities of traits and QTL densities ${ }^{1}$

\begin{tabular}{|c|c|c|c|c|c|c|c|c|c|c|}
\hline \multirow{4}{*}{$\begin{array}{l}\text { Initial } \\
\text { QTL } \\
\text { density }\end{array}$} & \multirow[b]{4}{*}{ Scenario $^{2}$} & \multicolumn{9}{|c|}{ Average annual genetic trends, in genetic SD units } \\
\hline & & \multicolumn{3}{|c|}{ Trait 1} & \multicolumn{3}{|c|}{ Trait 2} & \multicolumn{3}{|c|}{ Global breeding goal } \\
\hline & & & & & \multicolumn{3}{|c|}{ Initial heritabilities of traits $^{3,4}$} & \multirow[b]{2}{*}{$0.4 / 0.4$} & \multirow[b]{2}{*}{$0.4 / 0.2$} & \multirow[b]{2}{*}{$0.2 / 0.2$} \\
\hline & & $0.4 / 0.4$ & $0.4 / 0.2$ & $0.2 / 0.2$ & $0.4 / 0.4$ & $0.4 / 0.2$ & $0.2 / 0.2$ & & & \\
\hline \multirow{3}{*}{$\begin{array}{l}\text { 10QTL/ } \\
\text { Morgan }\end{array}$} & BLUP-AM & 0.36 & 0.37 & 0.36 & 0.20 & $0.15^{\mathrm{a}}$ & 0.16 & 0.40 & 0.37 & $0.37^{\mathrm{a}}$ \\
\hline & GE-1TP & 0.26 & 0.27 & 0.23 & 0.35 & $0.28^{\mathrm{b}}$ & 0.29 & 0.43 & 0.39 & $0.37^{\mathrm{a}}$ \\
\hline & GE-2TP & 0.39 & 0.40 & 0.41 & 0.34 & $0.27^{\mathrm{b}}$ & 0.26 & 0.52 & 0.47 & $0.48^{\mathrm{b}}$ \\
\hline \multirow{3}{*}{$\begin{array}{l}\text { 30QTL/ } \\
\text { Morgan }\end{array}$} & BLUP-AM & 0.52 & 0.53 & 0.49 & 0.20 & 0.14 & 0.14 & 0.50 & $0.47^{\mathrm{a}}$ & 0.45 \\
\hline & GE-1TP & 0.33 & 0.36 & 0.27 & 0.40 & 0.30 & 0.32 & 0.52 & $0.46^{\mathrm{a}}$ & 0.42 \\
\hline & GE-2TP & 0.57 & 0.60 & 0.58 & 0.35 & 0.25 & 0.25 & 0.65 & $0.60^{\mathrm{b}}$ & 0.58 \\
\hline \multirow{3}{*}{$\begin{array}{l}\text { 60QTL/ } \\
\text { Morgan }\end{array}$} & BLUP-AM & 0.60 & 0.62 & 0.55 & 0.19 & 0.13 & 0.14 & $0.56^{\mathrm{a}}$ & 0.54 & 0.49 \\
\hline & GE-1TP & 0.38 & 0.42 & 0.32 & 0.41 & 0.30 & 0.33 & $0.56^{\mathrm{a}}$ & 0.51 & 0.46 \\
\hline & GE-2TP & 0.68 & 0.71 & 0.67 & 0.35 & 0.24 & 0.25 & $0.73^{b}$ & 0.68 & 0.66 \\
\hline
\end{tabular}

\footnotetext{
${ }^{1}$ Results are averages of 200 replicates. composed of relatives for Trait 2 .

${ }^{3}$ Initial heritability for Trait $1 /$ initial heritability for Trait 2.

${ }^{4}$ For a given QTL density, all values within a column differ $(P<0.001)$ except values with common superscripts.
}

${ }^{2}$ Simulated scenarios: BLUP-AM = breeding scheme based on traditional BLUP-Animal Model genetic evaluations using phenotypes of the candidates for Trait 1 and phenotypes of relatives for Trait 2; GE-1TP = breeding scheme based on genomic evaluations using a single training population composed of relatives; GE-2TP = breeding scheme based on genomic evaluations using a training population composed of candidates for Trait 1 and a training population 
Table 4. Average annual increase in inbreeding among young candidates under 3 scenarios during the last $10 \mathrm{yr}$ simulated, according to the initial heritabilities of traits and QTL densities ${ }^{1}$

\begin{tabular}{lcccc}
\hline \hline & & \multicolumn{3}{c}{$\begin{array}{c}\text { Average annual increase } \\
\text { in inbreeding coefficient, } \%\end{array}$} \\
\hline Initial QTL & & Scenario $^{2}$ & \multicolumn{2}{c}{ Initial heritabilities of traits } \\
density & & $0.4 / 0.4$ & $0.4 / 0.2$ & $0.2 / 0.2$ \\
\hline 10QTL/ & BLUP-AM & +0.82 & +0.84 & +1.17 \\
Morgan & GE-1TP & +0.65 & +0.65 & +0.81 \\
& GE-2TP & +0.40 & +0.43 & +0.50 \\
\hline 30QTL/ & BLUP-AM & +0.90 & +0.79 & +1.10 \\
Morgan & GE-1TP & +0.65 & +0.68 & +0.77 \\
& GE-2TP & +0.39 & +0.38 & +0.45 \\
\hline 60QTL/ & BLUP-AM & +0.88 & +0.81 & +1.17 \\
Morgan & GE-1TP & +0.69 & +0.67 & +0.83 \\
& GE-2TP & +0.40 & +0.37 & +0.47 \\
\hline
\end{tabular}

\footnotetext{
${ }^{1}$ Results are averages of 200 replicates.

${ }^{2}$ Simulated scenarios: BLUP-AM = breeding scheme based on traditional BLUP-Animal Model genetic evaluations using phenotypes of the candidates for Trait 1 and phenotypes of relatives for Trait 2; GE-1TP = breeding scheme based on genomic evaluations using a single training population composed of relatives; GE-2TP = breeding scheme based on genomic evaluations using a training population composed of candidates for Trait 1 and a training population composed of relatives for Trait 2 .

${ }^{3}$ Initial heritability for Trait $1 /$ initial heritability for Trait 2 .

${ }^{4}$ For a given QTL density, the 3 values within a column differ $(P<0.001)$.
}

GE-2TP scenarios contained 1,000 animals phenotyped during the $4 \mathrm{yr}$ preceding the implementation of genomic evaluation, assuming that the DNA of these animals was available. In practice, pig breeding organizations have in recent years been building up DNA banks with the most important animals in their nucleus populations (including animals tested in central testing stations) to prepare for genomic research programs. Such an initial TP should therefore become available quite rapidly.

Conversely, for traits where a large quantity of phenotypes may be available and directly recorded on candidates, a breeding scheme based on the BLUP-AM methodology is already highly efficient. In the present study, the GE-2TP scenario only outperformed the BLUPAM scenario by 8 to $22 \%$ with respect to genetic trends for Trait 1 , despite the fact that the TP contained several tens of thousands of animals. By contrast, a breeding scheme replacing the phenotyping of candidates with a TP of limited size (as under the GE-1TP scenario) would clearly be less efficient for field data. To conclude, GS should not be substituted for the direct phenotyping of candidates when the latter is possible.

Under the present simulations, TP were updated only once a year by adding relatives (GE-1TP and GE2TP scenarios) and candidates (GE-2TP) phenotyped during the current TS and previous 17 TS. Consequently, whereas candidates available for selection in the TS in which the SNP effects were estimated or reestimated had closely related animals in the TP (sibs or half sibs) or were

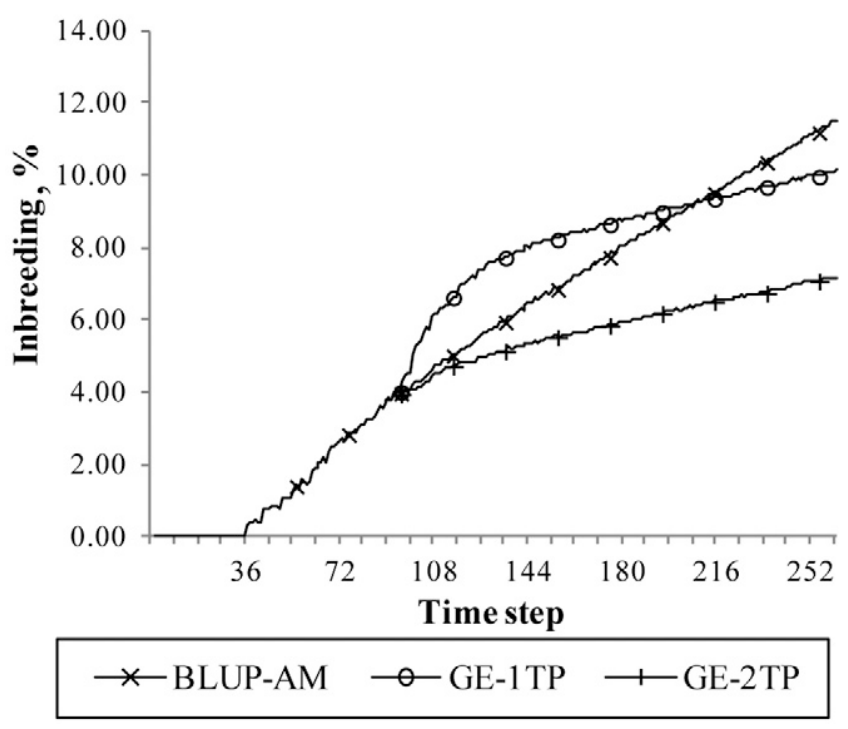

Figure 7. Evolution of inbreeding among young candidates under 3 scenarios during the $15 \mathrm{yr}$ simulated. Initial QTL density $=60 \mathrm{QTL} / \mathrm{M}$ for each trait, initial heritability of Trait $1=0.4$, and initial heritability of Trait $2=0.2$. The results are averages of 200 replicates. BLUP-AM = breeding scheme based on traditional BLUP-Animal Model genetic evaluations using phenotypes of the candidates for Trait 1 and phenotypes of relatives for Trait 2; GE-1TP = breeding scheme based on genomic evaluations using a single training population composed of relatives; GE-2TP = breeding scheme based on genomic evaluations using a training population composed of candidates for Trait 1 and a training population composed of relatives for Trait 2 .

even themselves part of the TP (for Trait 1 under the GE2TP scenario), candidates in the next $17 \mathrm{TS}$ were less and less closely related to this TP because of the continuous renewal of breeding animals. The progressive annual decrease in the accuracy of GEBV observed between 2 consecutive reestimates of the SNP was therefore consistent with the findings of several other studies (e.g., Solberg et al., 2009; Habier et al., 2010; Wolc et al., 2011), which showed that GEBV precision decreases in line with the degree of relationship between the TP and the population under evaluation, thus confirming the importance of continuously renewing the TP.

However, the reason for the global slowdown in the increase of GEBV accuracy and for the decrease after TS144 for Trait 1 under the GE-2TP scenario was different. As shown in Eq. [1], the accuracy of GEBV depends on the heritability of the trait, which decreased over time during the simulations because of the increase in the incidence of favorable QTL alleles, thus reducing the genetic variance of the traits. Consequently, the impact of adding new animals to the TP became weaker, and the rate of increase in accuracy gradually decelerated. In the case of Trait 1 under the GE-2TP scenario, where the TP size remained constant after TS144, renewal of the TP by replacing the 13,770 oldest animals with 13,770 more recent ones did not compensate for the loss of power due to less heritability, and the accuracy of the GEBV for this trait eventually decreased. 
The observed decrease over time in the genetic variance for both traits was actually very marked, whichever methodology was used to estimate breeding value. For example, only 27 and $35 \%$ of the initial variance for Trait 1 remained under the GE-2TP and BLUP-AM scenarios after $15 \mathrm{yr}$ of selection in the $\mathrm{MLh}^{2}$-HighQd case, these values decreasing to 8 and $12 \%$, respectively, in the $\mathrm{MLh}^{2}$-LowQd case. The overall decrease in the genetic variance was less for Trait 2, because the EBV were globally less accurate than for Trait 1, resulting in a smaller Bulmer effect and a less efficient fixation of the favorable QTL alleles for Trait 2.

Our study helps alleviate the paucity of literature with respect to the evolution of genetic variance in GS breeding schemes. A major decrease in the genetic variance and accuracy of the EBV was reported by Jannink (2010) in a stochastic simulation of GS in barley (Hordeum vulgare). Nielsen et al. (2011) also observed a great decrease in genetic variance after 10 generations of stochastic simulation of a fish breeding scheme based either on BLUP or genomic evaluations. However, the decrease in genetic variance was less than in the present study because of lower selection rates, larger initial number of QTL and inbreeding management to restrain the inbreeding rate per generation to 0.5 or $1 \%$.

The evolution of genetic variance observed here during the early years after implementation of selection was consistent with what could be expected according to the Bulmer effect (Dekkers, 1992). Nevertheless, the magnitude of the long-term decrease exceeded the theoretical predictions based on the infinitesimal polygenic model. Moreover, this loss of genetic variability constituted a deviation from what might be observed in true populations selected for tens of years, where genetic diversity is still not exhausted. Nevertheless, we simulated a finite number of QTL at the beginning of the simulation, and as the number of segregating QTL decreased because of selection, our model diverged more and more from the polygenic infinitesimal model underlying the theoretical formulas.

No mutation process was implemented in our simulations. However, mutations are not expected to impact the results within the first 30 to 40 generations of selection (Hill, 1985) whereas our horizon of interest was limited to $15 \mathrm{yr}$ of pig breeding (equivalent to about 10 generations of selection). As a result, the most likely hypothesis is that the large reduction in variance observed here was due to the very high intensities of selection performed on male and female pathways, and to the finite number of QTL segregating at the beginning of the simulation. Considering a much larger number of QTL initially segregating in the population as did Lillehammer et al. (2011) and Nielsen et al. (2011) who simulated 1,800 and 1,000 QTL, respectively, might be a solution to preserve genetic variability. A dynamic modeling of gene effects, taking account of interactions between loci, might pave the way to exploring the preservation of genetic variability. Finally, considering the relationships at selection and mating steps, or even adding a constraint on the inbreeding rate as made by Nielsen et al. (2011), could also slow the decrease in genetic variance.

Our results concerning the GE-2TP and BLUPAM scenarios confirm the theoretical expectations of Daetwyler et al. (2007) that GS should reduce the rate of inbreeding per generation when compared with selection based on traditional BLUP-AM EBV. The breeding value of an individual can be conceived as the combination of the breeding values of its parents and a Mendelian sampling term. The Mendelian sampling term is poorly estimated with the BLUP-AM methodology, the phenotypes of ancestors and relatives mostly contributing to estimating the parental component of a breeding value. In particular, full-sib candidates obtain exactly the same BLUP-AM EBV for traits exclusively recorded on relatives (i.e., Trait 2 in the present study). Consequently, the BLUP-AM scenario favored the co-selection of related animals among candidates. By contrast, under the GE-2TP scenario, the genotypes at markers enabled a more precise estimate of the Mendelian component of breeding values for Traits 1 and 2, resulting in fewer correlated EBV among sibs and more accurate EBV for each of them.

The sharp initial rise in the inbreeding rate under the GE-1TP scenario might have appeared inconsistent at first sight. Nevertheless, as shown by Habier et al. (2010), the GEBV accuracy of an animal is strongly dependent on its relationship with the TP. When genomic evaluation was implemented at TS90 under the GE-1TP scenario, the TP was limited to 1,000 animals sampled from only $10 \%$ of the litters born during the 4 previous years, which resulted in a considerably smaller average accuracy for Trait 1, when compared with the BLUP-AM scenario where all candidates were phenotyped, and compared with the GE-2TP scenario where all candidates were closely related to the TP for Trait 1 . One can therefore hypothesize that the candidates belonging to the few families most closely related to the TP had a greater chance of being selected, because their more accurate GEBV were less regressed toward the mean. This would explain the greater initial inbreeding rate. Thereafter, as the TP size and the average relationship between the candidates and TP increased, the co-selection of related candidates decreased and the increase in inbreeding slowed. Further work, however, has to be done to better understand this phenomenon.

It may seem inconsistent that the GE-2TP scenario produced the smallest increase in the average inbreeding 
rate while at the same time causing the most rapid reduction in genetic variance. In fact, the GE-2TP scenario produced the greatest genetic trends because of the strong accuracy of its EBV, resulting in more rapid fixing of favorable alleles of the QTL. As mentioned above, the co-selection of related animals is small in a highly accurate genomic breeding scheme. Consequently, a larger proportion of the homozygosity observed in the population results from selection of identity-by-state loci in GE-2TP compared with BLUP-AM and GE-1TP. Therefore, the pedigree-based inbreeding measurement, which only takes into account the identity-by-descent part of the population homozygosity, does not fully reflect the genetic diversity of the population.

No attention was paid in the simulations to inbreeding management in the selection and mating procedures whereas limitation of the inbreeding rate is an important concern for pig breeders. As mentioned by Lillehammer et al. (2011), adding a constraint on this parameter would probably have reduced the inbreeding rate, but might also have affected genetic trends under the BLUP-AM scenario. The superiority of GE-2TP over BLUP-AM would therefore be even greater with respect to genetic gains in the context of a constant inbreeding rate across the scenarios.

In light of these results, one might ask if it would be economically profitable to move from a classical pig male line breeding scheme to a genomic breeding scheme organized as under the GE-2TP scenario. As an initial response to this question, one may consider a very simple breeding goal combining ADG and feed efficiency, representing Traits 1 and 2 of the simulations, respectively. The GE-2TP scenario resulted in additional genetic gains of $+0.09 \sigma_{g} / \mathrm{yr}$ for Trait 1 and $+0.11 \sigma_{g} / \mathrm{yr}$ for Trait 2 when compared with the BLUP-AM scenario (HighQd $\times \mathrm{MLh}^{2}$ case). Considering that half of the genetic improvement achieved in the selection nucleus is transmitted to the 15,000,000 pigs finished in France each year and bred by a purebred Piétrain sire, the estimated increase in profit in production herds due to GS would be approximately $€ 1,300,000 / y$ r. Assuming that the costs induced by creating the 2 initial TP would be written off within $4 \mathrm{yr}$, the cost of genotyping should not exceed approximately $€ 140$ per animal, so that the extra profits due to increased genetic gains in production would compensate for the annual genotyping costs of 13,770 new candidates and 270 animals increasing the TP for Trait 2. The break-even cost, however, would depend on the size of the nucleus population and on its extent at the production level. For example, the breakeven cost of genotyping in the same terminal male line with only 10,000,000 finished offspring per year would be approximately $€ 100$ per animal.
A comprehensive economic study considering a more realistic breeding goal and appropriate economic weights is obviously needed to reach a firm conclusion, but this rough estimate suggests that the implementation of genomic evaluation in a pig male line is economically feasible. The porcine $60 \mathrm{~K}$ BeadChip is currently expensive, but the price will almost certainly decrease in the future as happened in the case of cattle in recent years. Another way to reduce costs would be to genotype candidates with a less expensive lower density array and use genotype imputation techniques to preserve the efficiency of genomic evaluation, following the example of dairy cattle (Dassonneville et al., 2011; Wiggans et al., 2012).

Finally, it would also be useful to quantify the increase in genetic gain that might be achieved if the additional cost of implementing genomic evaluation were invested in improving the current BLUP-AM breeding scheme, for example, by increasing phenotyping capacity for relatives. A constant cost comparison of the results of alternative breeding schemes would aid decision making regarding the economic value of GS.

\section{LITERATURE CITED}

Daetwyler, H. D., B. Villanueva, P. Bijma, and J. A. Woolliams. 2007. Inbreeding in genome-wide selection. J. Anim. Br. Genet. 124:369-376.

Daetwyler, H. D., B. Villanueva, and J. A. Woolliams. 2008. Accuracy of predicting the genetic risk of disease using a genome-wide approach. Plos One 3:1-8.

Dassonneville, R., R. F. Brøndum, T. Druet, S. Fritz, F. Guillaume, B. Guldbrandtsen, M. S. Lund, V. Ducrocq, and G. Su. 2011. Effect of imputing markers from a low-density chip on the reliability of genomic breeding values in Holstein populations. J. Dairy Sci. 94:3679-3686.

Dekkers, J. C. M. 1992. Asymptotic response to selection on best linear unbiased predictors of breeding values. Anim. Prod. 54:351-360.

Habier, D., J. Tetens, F. R. Seefried, P. Lichtner, and G. Thaller. 2010. The impact of genetic relationship information on genomic breeding values in german holstein cattle. Genet. Sel. Evol. 42:5.

Hayes, B., and M. E. Goddard. 2001. The distribution of the effects of genes affecting quantitative traits in livestock. Genet. Sel. Evol. 33:209-229.

Hayes, B. J., P. J. Bowman, A. J. Chamberlain, and M. E. Goddard. 2009. Genomic selection in dairy cattle: Progress and challenges. J. Dairy Sci. 92:433-443.

Hill, W.G. 1985. Effects of population size on response to short and long term selection. J. Anim. Br. Genet. 102:161-173.

Jannink, J. L. 2010. Dynamics of long-term genomic selection. Genet. Sel. Evol. 42:35.

Lillehammer, M., T. H. E. Meuwissen, and A. K. Sonesson. 2011. Genomic selection for maternal traits in pigs. J. Anim. Sci. 89:3908-3916.

Meuwissen, T. H. E. 2009. Accuracy of breeding values of 'unrelated' individuals predicted by dense SNP genotyping. Genet. Sel. Evol. 41:35.

Meuwissen, T. H. E., B. J. Hayes, and M. E. Goddard. 2001. Prediction of total genetic value using genome-wide dense marker maps. Genetics 157:1819-1829. 
Muir, W. M. 2007. Comparison of genomic and traditional BLUPestimated breeding value accuracy and selection response under alternative trait and genomic parameters. J. Anim Breed. Genet. 124:342-355.

Nielsen, H. M., Sonesson A. K., and T. H. E. Meuwissen. 2011. Optimum contribution selection using traditional best linear unbiased prediction and genomic breeding values in aquaculture breeding schemes. J. Anim. Sci. 89:630-638.

Quaas, R. L., and E. J. Pollak. 1980. Mixed model methodology for farm and ranch beef cattle testing programs. J. Anim. Sci. 51:1277-1287.

Solberg, T. R., A. K. Sonesson, J. A. Woolliams, J. Degard, and T. H.
E. Meuwissen. 2009. Persistence of accuracy of genome-wide breeding values over generations when including a polygenic effect. Genet. Sel. Evol. 41:53.

Wiggans, G.R., T.A. Cooper, P.M. VanRaden, K.M. Olson, and M.E. Tooker. 2012. Use of the Illumina Bovine3K BeadChip in dairy genomic evaluation. J. Dairy Sci. 95:1552-1558.

Wolc, A., J. Arango, P. Settar, J. E. Fulton, N. P O’Sullivan, R. Preisinger, D. Habier, R. Fernando, D. J Garrick, and J. C. M. Dekkers. 2011. Persistence of accuracy of genomic estimated breeding values over generations in layer chickens. Genet. Sel. Evol.43:23. 
References

This article cites 18 articles, 3 of which you can access for free at: http://www.journalofanimalscience.org/content/90/12/4164\#BIBL 